Volumen: 23 Fecha de recibido: 27/10/2020 Fecha de publicación: Enero-Junio de 2021

Año: 2021 Fecha de aceptado: 7/12/2020 Correo: revista.societas@up.ac.pa

Numero: 1 Número de Páginas: 93-110 URL:https://revistas.up.ac.pa/index.php/societas

\title{
La Oscuridad En Medio De La Opulencia. San Miguelito Donde "No Llega EI Sol Porque Es Aristocrático" Una Visión histórica Social De La Realidad Del Recurso Humano.
}

The Darkness In The Midst Of Opulence. San Miguelito Where "The Sun Does Not Arrive Because It Is Aristocratic" A Historical Social Vision Of The Reality Of The Human Resource.

José B. Álvaro P. copedomme@yahoo.es

Kayrha Sánchez, kayrha sanchez@yahoo.es

Nancy Castillo, prof.nancy30@gmail.com

Florencio Muñoz, fr-munozb@outlook.es

Aris Pitty, zanedv@hotmail.com

Universidad de Panamá, Panamá

\section{Resumen}

San Miguelito es una comunidad enclavada en el corazón de la capital de la República de Panamá. Nació hace 70 años, cuando sus primero pobladores fueron empujados por las necesidades sociales sin lo más elemental para sobrevivir: sin agua, sin luz, sin viviendas (si pudiera así llamársele) forrada con zinc, cartón o madera desechable. Concentra hoy el 10\% de la población de la Republica. Allí todavía llegan los migrantes de todos los rincones de país. Es un mosaico de las diferentes expresiones culturales del país y aun refleja las profundas necesidades de los marginados sociales. De esa desventajosa condición social se aprovechan los demagogos políticos. Concentra un alto porcentaje de delincuencia y prostitución, ausencia de un transporte adecuado, desnutrición, alto desempleo y subempleo. En el presente la voracidad del capital inmobiliario ha usurpado terreno y es notable el contraste entre los sectores de la comunidad desposeídos y los habitantes recién llegados. Los últimos son familias profesionales de ingresos económicos medios a altos. El presente escrito trata de explicar las causas de esta tragedia.

Palabras clave: estructura social, transformación de la tierra, teoría del espacio, modelo de producción capitalista, asentamientos humano.

\section{Abstract}

San Miguelito is a community nestled in the heart of the capital of the Republic of Panama. It was born 70 years ago, when its first inhabitants were pushed by social 
needs without the most basic elements to survive: without water, without electricity, without housing (if it could be called that) lined with zinc, cardboard or disposable wood. Today it concentrates $10 \%$ of the population of the Republic. Migrants from all corners of the country still arrive there. It is a mosaic of the different cultural expressions of the country and still reflects the deep needs of the socially marginalized. Political demagogues take advantage of this disadvantageous social condition. It concentrates a high percentage of crime and prostitution, lack of adequate transportation, malnutrition, high unemployment and underemployment. At present, the voracity of real estate capital has usurped ground and the contrast between the dispossessed sectors of the community and the newly arrived inhabitants is remarkable. The latter are professional families of medium to high income. This writing tries to explain the causes of this tragedy.

Keywords: social structure, land transformation, space theory, capitalist production model, human settlements.

\section{Introducción}

\section{Caracterización de los espacios en la zona de transito de Panamá al interés} del capital global

La construcción del ferrocarril inició el proceso de incorporación del istmo de Panamá a la economía capitalista mundial robustecido con la construcción del Canal Interoceánico a inicio del siglo XX. La producción abundante de mercancía obliga a los centros hegemónicos, particularmente los Estados Unidos a la búsqueda de nuevas áreas periféricas donde colocar sus productos y luego reexportarlos.

La revolución de los medios de transporte impulsaría la modernización de la ruta, y a la vez produciría modificaciones en la evolución de nuevas formas de concentración de la población, respondiendo a la lógica de las relaciones de producción imperantes, variando el papel y uso de los espacios.

Sustentado en lo anterior, el desarrollo teórico permite establecer como se incorpora el espacio a un modelo de producción capitalista determinado por las necesidades históricas del sistema de reestructurar su función a la lógica del capitalismo global. 
La economía política define el espacio, como un subproducto social del modo de producción asociado al papel que el territorio desempeña en el mundo capitalista. Las transformaciones y la configuración espacial capitalista están directamente relacionada con los ciclos de auge y depresión de éste y de las necesidades de acumulación planteadas en cada una de sus fases de desarrollo. Dada su naturaleza, el sistema tiende a emplear grandes cantidades de capital fijo que no pueden reubicarse fácilmente, emergiendo una de sus contradicciones, pues el sistema deposita en el capital fijo la confianza para incrementar el valor de la productividad del trabajo y encuentra que su calidad de fijo e inmóvil se convierte en una barrera que hay que superar. Esta es la base para entender los procesos de la formación y resolución de las crisis de la economía espacial capitalista: la concentración de capital propicia su devaluación, así en el curso de las crisis "se libera" capital para establecer nuevas tecnologías y nuevas estructuras espaciales relocalizando la producción o incorporando nuevos espacios económicos.

\section{La Relación De La Especie Humana Y Su Interacción Con EI Espacio Fisico}

Los efectos de las relaciones humanas sobre el espacio eran perceptibles en antelación con la presencia de los Estados Unidos en Panamá. Se evidenció en la devastación de considerables zonas boscosas al Noreste de la ciudad de Panamá para dar paso a las sabanas y a un conjunto de actividades agropecuarias, como el pastoreo, la producción bananera, la producción de azúcar, cacao, café y caucho y la horticultura en poblados ubicados en lo que hoy conocemos como área canalera.

La transformación del paisaje natural a un contexto sociocultural permitió establecer otras formas de explotación económica. De esta manera surgió la agrosilvicultura, actividad económica de corte familiar de auto subsistencia, estructuradas en fincas de pocas hectáreas que, a su vez, constituía el hábitat de sus integrantes, quienes establecieron un sistema de vida satisfactoria en medio de espacios frutales y vegetales suficientes para satisfacerlas, sin preocupaciones de carencia de los recursos comestibles. 
Justamente, la existencia de ciclos de auge y depresión que determinan diferentes fases del sistema, hacen posible que un espacio mantenido en reserva cambie su estatus como territorio estratégico al ofrecer una serie de ventajas requeridas por el modelo de acumulación. Veamos los elementos analizados para comprender la configuración de los cambios que experimenta un territorio estratégico para la expansión capitalista.

"Los cambios en el medio físico delinearon una nueva condición contractual incluyendo las estructuradas por los pobladores de importantes zonas de la línea de la construcción del canal quienes tuvieron que abandonar su sitio de vivienda y sus actividades económicas. Ejemplo de ello son los cambios experimentados en el valle del Chagres, convertido en un lago artificial de 268 kilómetros cuadrados - "con dimensiones similares a isla de Barbados -Acontecimiento que sumergió poblados pintorescos y productivos como Gatún y Matachín"

El espacio construido constituye el testigo de las tensiones entre los elementos de la estructura social, ante todo es un producto social y la teoría del espacio solo puede concretarse a través de la estructura social. De ahí que sea no solo sobre el terreno que el geógrafo deba buscar la clave explicativa de la forma de organización especial.

La dinámica se repite ante la necesidad de una nueva represa en la parte superior del Chagres, en el área conocida como Villa de Alhajuela, al noreste de Gamboa, para el almacenaje del recurso hídrico durante el periodo Iluvioso, con el fin de mantener el lago Gatún "en nivel estable todo del año". El proceso de devastación continúa con la obtención de nuevas extensiones de tierra, lo cual hace imperativo, que entre 1940 y 1942, la construcción de la primera carretera en la región Transístmica del Istmo que unificó las provincias de Panamá y Colón.

Guillermo Castro (2010) sostiene que entre las alteraciones de orden social y cultural asociadas a la construcción del Canal están la desaparición de paisajes que habían desempeñado un importante papel en la forja de nuestra 
identidad histórica, como el gran valle del Chagres, con sus selvas, sus poblados, sus pastizales y sus cultivos de banano - descritos por Gil Blas Tejiera en su novela Pueblos Perdidos, (1962) a esto se agregó la devastación de la cuenca del río Grande -en cuya desembocadura fue ubicado el vertedero de La Boca.

Lo anterior explica las nuevas relaciones sociales, culturales y naturales con el surgimiento de un frente de colonización agropecuaria, un centro urbano industrial y, asimismo, nuevos procesos de deforestación en la cuenca media del Chagres, detenido gracias a la creación de los parques nacionales de Chagres, Camino de Cruces y Soberanía en la década de 1980. (Farnum y Murillo, 2015).

Thomas Kuhn (1971) inscribe este proceso dentro de los parámetros de la ciencia cuando nos dice que esta no la reduce a la mera acumulación de hecho, sino que se aplica a la organización de tales hechos en un tipo de paradigmas o modelo explicativo del funcionamiento de la naturaleza. Los viejos paradigmas pierden su atractivo, y otros nuevos surgen para ocupar su lugar.

Donald Worster (2006) en su enfoque bibliográfico sobre transformación de la tierra es del criterio que existe una conexión entre los paradigmas superados y los emergentes, al expresar los científicos, no trabajar en completo aislamiento respecto a sus sociedades: por el contrario, reflejan en sus modelos la naturaleza de tales sociedades, sus modos de producción, sus relaciones humanas, y las necesidades y valores propias de su cultura.

\section{La Formación Social A La Luz Del Modelo De Producciòn Capitalista.}

"En la producción social de su vida, los hombres contraen determinadas relaciones necesarias e independientes de su voluntad, relaciones de producción, que corresponden a una determinada fase de desarrollo de sus fuerzas productivas materiales. El conjunto de estas relaciones de producción forma la estructura económica de la sociedad, la base real sobre la que se levanta la superestructura jurídica y política y a la que corresponden determinadas formas de 
conciencia social". (Álvaro Sevilla, 2012) El modo de producción de la vida material condiciona el proceso de la vida social, política y espiritual en general.

Para lograr su desarrollo, el capitalismo tuvo que otorgarse una economía política, penetrar las redes de poder formal y adueñarse de sus estructuras, construir nuevos aparatos de gobierno del cambio social y artículos en un núcleo institucional directivo (Habermas, 1985).

Castillo Jorge (1986) al analizar la formación social la enmarca enfatizando el modo de producción que a su juicio responden a un determinado grado de desarrollo de las fuerzas productivas, de las sociedades históricamente determinadas, la producción de bienes materiales no ocurre de una forma homogénea, sino que en una misma sociedad se encuentran distintas relaciones de producción y por lo tanto, distintos niveles de desarrollo de las fuerzas productivas. Una sociedad puede estar comprendida por diferentes modos de producción que no actúan aisladamente, sino que están interrelacionadas entre las instancias de los distintos modos de producción.

Lo que Carlos Marx (1998) al analizar la acumulación originaria del capitalismo la estableció como "el proceso que engendra el capitalismo sólo puede ser uno: el proceso de disociación entre el obrero y la propiedad sobre las condiciones de su trabajo, proceso que de una parte convierte al capital en los medios sociales de vida y de producción mientras de otra parte convierte a los productores directos en obreros asalariados .La llamada acumulación originaria no es más que el proceso histórico de disociación entre el productor y los medios de producción.

El concepto modo de producción que se refiere a un modelo abstracto, la formación social se acerca más a una realidad concreta compleja. De esta manera, puede ser considerada como una totalidad social, históricamente determinada, estructurada a partir de la forma en que se combinan las diferentes relaciones de producción que existen a nivel de la estructura económica y que se repiten de forma igual, en la medida en que no frenen el desarrollo de las fuerzas 
productivas. Castillo (1986) define formación social como el lugar dinámico en el que puede captarse el desarrollo de las fuerzas productivas cuyo peso marca las relaciones de producción.

Lógica de la estructura económica o instancia dominante y determinante a lo planteado en el prólogo a la contribución a la critica de la economía política por Carlos Marx (1998) cuando designa al conjunto estructurado de las relaciones de producción con la expresión estructurada económica de la sociedad y las relaciones con la totalidad de los fenómenos de conciencia social al señalar: "El conjunto de estas relaciones de producción constituyen la estructura económica de la sociedad, la base real sobre la cual se eleva una superestructura, jurídica y política y a la que corresponden formas sociales determinadas de conciencia. El concepto de superestructura define dos niveles de la sociedad: la estructura jurídica política a la cual corresponde el Estado y el Derecho y la estructura ideológica, la que implica las formas de conciencia social".

"El modo de producción es un concepto teórico abstracto que nos permite conocer en forma científica una totalidad social estructurada a partir de diferentes instancias (económicas, políticas, e ideológicas), que juegan un papel distinto en la sociedad donde una instancia puede ser la dominante, pero la instancia económica, será determinante.

\section{Desarrollo}

\section{La Economía Panameña Y El Desarrollo Del Proceso De Sustitución De Importaciones Y EI Surgimiento Del Distrito De San Miguelito.}

La finalización de la Segunda Guerra Mundial dinamizó el desarrollo de la economía panameña, fundamentalmente la industria de los alimentos (arroz, leche, café, y tomate), así como el calzado y los vestidos; ese modelo conocido como sustitución de importaciones, marcó modificaciones sociales, culturales, y económicas en el espacio conocido hoy, como distrito de San Miguelito, ocupación determinada por el proceso de migración proveniente de las áreas rurales, principalmente de la península de Azuero y Veraguas; otros procedentes de "asentamientos humanos de áreas ribereñas del Canal, quienes producto de la 
construcción de la vía acuática, fueron empujados a trasladarse, a lugares, en aquellos instantes rurales, como Cerro Viento, Santa Pera, las Trancas, Ciudad Jardín San Antonio (Montoya, 1997, pág. 45). Quedaba concentraba las fuerzas productivas cercanas a los centros de producción.

Hacia 1950, los 51 kilómetros cuadrados del distrito de San Miguelito, su propiedad territorial, estaba monopolizada por cinco familias cuyos propietarios constituían parte de la médula del poder económico y político del país. Sobresalían las familias Carbone, Recuero, Fábrega, Harmodio Arias Madrid, y los Goytía, obligadas por la presión popular y la iglesia católica tuvieron que aceptar la indemnización ofrecida por las autoridades gubernamentales. Empezó la transformación de su entorno y espacio. En realidad la estructura de la tenencia de la tierra presagiaba enfrentamientos sociales de incalculables consecuencias. Eran cotidianos las hostilidades por las necesidades básicas de sus habitantes.

Lo que Henri Lefebvre (1974) denominó concepto de "producción del espacio" retomando el sentido marxista de producción para constituir el universal concreto, una realidad social, un conjunto de relaciones y formas. Así, el espacio social no sería una cosa entre otras cosas, sino que envuelve las cosas producidas, comprende sus relaciones de coexistencia y simultaneidad, orden y desorden relativo, razón por la cual no puede reducirse a simple objeto.

El desarrollo teórico permite establecer un planteamiento que surge del modelo de producción capitalista cuyo origen y evolución está determinado por las necesidades históricas del sistema $\mathrm{y}$, particularmente, por el proceso de reestructuración de mediados del siglo XIX, y que Incorpora este tipo de espacios en la etapa del capitalismo global.

\section{Características Políticas Económicas Y Físicas Del Distrito De San Miguelito.}

Su ocupación, como asentamiento humano, carente de políticas articuladas, acciones interdisciplinarias e interinstitucionales a través del gobierno local ha impedido establecer alianzas necesarias que optimice el capital humano; ausente de planificación en aras para solucionar los problemas 
de la comunidad, dentro de los parámetros de la Gobernabilidad y Procesos Democráticos; Económicos y Desarrollo; Seguridad Alimentaria y Participación Social; y Educación, Salud y Ambiente.

Hoy, el llamado gobierno local se ha convertido en un centro político de empleo, recurso humano poco profesional, cuyo objetivo mas que solucionar necesidades sociales apremiantes es graficar el clientelismo político

Las características climáticas presentan una precipitación anual de aproximadamente 2,000 m.m.; su humedad relativa es de $75 \%$ y la temperatura oscila entre 27 y 35 grados centígrados, como resultado de las masas oceánicas y la angosta franja que lo separa los oceános. Desde el punto de vista geomorfológico, se caracteriza por aéreas onduladas, montañas con elevaciones de 30 a 200 metros que determinan el uso y aprovechamiento del suelo. De acuerdo a cifras suministrada por la Dirección de Estadística y Censo de la Contraloría General de la República, durante la década 60, del siglo pasado, la población de San Miguelito, ascendía a 13,000 habitantes con una densidad de 254 personas por kilómetro cuadrado; seis años después, en 1970 la población alcanzó guarismo de 68,400personas y la densidad se incrementó a 1368 personas por kilómetro cuadrado; transcurrido una década la población del distrito se eleva a 156,661 habitante y la densidad por kilómetros cuadrado fue de 3,132 personas; en 1990 la población se incrementó a 243,025 y el número por kilómetro cuadrado fue de 4,860 personas, El último censo del siglo XX, los dígitos de San Miguelito alcanzaban una población de 293,374 y su densidad de 5,867 personas por kilómetro cuadrado. Por último, el primer censo del nuevo milenio reveló la existencia de una población de 315,019 y la densidad de 6,300 personas por kilómetro cuadrado.

El incremento del movimiento migratorio, alcanzado en San Miguelito, es de $7.2 \%$ y es el resultado de la movilidad nacional e internacional, es sólo comparable a los distritos de Panamá, 24.3\%, y Colón de $18.7 \%$, representando el $18 \%$ de la de la población del distrito de Panamá y el $10 \%$ de la población total, distribuida político-administrativa en nueve corregimientos: 
Amelia Denis de Icaza, Belisario Porras, José Domingo Espinar, Mateo Iturralde, Victoriano Lorenzo, Belisario Frías, Omar Torrijos, Arnulfo Arias y Rufina Alfaro.

La situación socio económica del distrito surgida de la relación capital trabajo nos indica que el $60 \%$ de la población labora en el sector privada, un $17 \%$ en Entidades gubernamentales. El poder adquisitivo del 16\% proviene de las actividades informales; el $44.8 \%$ de la población devenga salarios por debajo del salario mínimo y el ingreso económico del $24.8 \%$ están entre $\mathrm{B} / .400 .00$ y B/.999.00.

Esta situación explica por qué la mitad de la población se encuentra por debajo de la línea de la pobreza, fenómeno que ilustra la fragilidad del núcleo familiar, la espiral de violencia, la deserción escolar, los embarazos precoces, la prostitución en niños (as) y en jóvenes adolescentes, la mendicidad infantil, la promiscuidad, el consumo de alcohol y drogas a temprana edad y la violencia intrafamiliar, la venta y el tráfico de armas, drogas, los homicidios, el sicariato, los asaltos a mano armada, robos y hurtos, entre otros.

Agregamos a ese cúmulo de carencias un sistema inadecuado de tratamiento de aguas servidas, la contaminación de las aguas superficiales producto de los desechos orgánicos, sustancias químicas inorgánicas y otros agentes contaminantes, constituye un grave problema ambiental, reduciendo la disponibilidad de los recursos hídricos, afectando el desarrollo de actividades domésticas, agropecuarias y recreativas; poca disponibilidad de agua para el consumo humano.

El deterioro de sus principales ríos lo demuestran los estudios efectuados por la Universidad de Panamá y la Universidad Tecnológica de Panamá; los mismos revelan un gran deterioro con elevadas concentraciones de materia orgánica y alta carga bacteriana, azotados por 165 sistemas de tratamiento de aguas negras, lo cual revela precarios niveles de salubridad individual y colectiva en que vive la población del distrito, sobre todo, el grupo más vulnerable: los 
niños. Ello explica el espiral de enfermedades infecto-contagiosas (meningitis, cólera), situación que se agudiza aún más con los niveles de pobreza, desempleo, sub empleo, bajos ingresos (inferiores al salario mínimo) que experimenta la población que, a su vez, repercute en el deterioro del medio ambiente y su entorno y, por añadidura, a la depauperación económica y social de sus habitantes.

La estructura social tiene una interacción directa entre el medio y la naturaleza. En el caso del distrito de San Miguelito encontramos prácticas de carácter culturales y patrones de conductas traídas desde sus lugares de origen que son reproducidas impactando el nuevo entorno. La carencia de ciertos conocimientos respecto a la norma urbana incide sobre ciertas viviendas edificadas sin contemplar la línea de construcción, agravándose con la ausencia de aceras y de áreas recreativas y culturales para la convivencia social.

\section{El Espacio Del Distrito De San Miguelito Y La Nueva Lógica Del Capital Inmobiliario.}

La concepción del "espacio" es el resultado del tránsito de diversas disciplinas a través de prácticas y reflexiones ideales del "espacio material" hasta la del "espacio como producto", determinado por prácticas sociales y productivas relacionadas con diferentes estadios del capital.

Desde una perspectiva del modelo económico de mercado, delineado a partir de la ideología neoliberal consistente en desregular del control estatal, la economía, flexibilización del mercado del trabajo, entre otros.

Marco Valencia lo denomina como un fenómeno que ha conformado las bases originales de las transformaciones territoriales, espaciales, y sociales de la ciudad, incidiendo directa o indirectamente en la desarticulación de la institucionalidad urbanística que antecede a la revolución neoliberal, concepción que con el transcurrir de los últimos treinta años ha variado ocurriendo como resultado del proceso global de la función del espacio a la luz del capital inmobiliario y su perspectiva de desarrollo del territorio, acentuando nuevas 
formas de crecimiento especulativo basado en posibilidades de movilidad rápida sustentad en las nuevas tecnologías y la organización de circulación de valores.

Los territorios urbanos de San Miguelito, hoy por hoy constituyen nuevos espacios de interés al desarrollo global de capital inmobiliario que deben dar a nuevos conglomerados $\quad$ nodos de circulación de mercancías y espacios sociales configurado dentro de los ajustes diseñado por el neoliberalismo, sobresaliendo en los espacios de dos de sus corregimientos utilizados que en el pasado se caracterizaron por el desarrollo de actividad agropecuaria, reorientado su función económica a partir de la década de los ochenta dirigida a la actividad, inmobiliaria de nivel medio y alto que oscilaban entre los treinta a cien mil , y quinientos mil Balboas.

Josep. M. Naredo siguiendo el argumento de Weber, Geddes y Braudel traduce la realidad espacial como las estructuras funcionales del régimen de acumulación que requiere la organización reticular del espacio en base a nodos, nódulos o densificaciones (que son los distintos organismos capaces de acumular: regiones, ciudades, empresas, etc.) interconectados por líneas de conexión funcional, que permiten la explotación de los recursos, necesarios en donde se encuentran, para llevar a los nodos y redistribuirlos una vez recombinados, tarea que implica la circulación 0 el transporte de flujos (recursos naturales 0 energéticos, materiales, etc.) necesarios para su combinación en los nodos.. Desde esta perspectiva, la ciudad se convierte en una gran máquina que extiende sus tentáculos depredadores a otros territorios en los cuales materializa sus funciones de apropiación y vertido; para lo cual necesita también convertirse en una máquina de dominio militar, político, cultural. Así lo demuestra la inyección de capitales realizadas en los últimos cinco años en San Miguelito, con la aprobación, como mínimo, de 10 proyectos millonarios de desarrollo urbano y comercial incluyendo la ampliación de nuevas etapas en San Antonio, Cerro Viento y Brisas del Golf, construcciones que requerirán una inversión de por lo menos 150 millones de dólares. 
Los procesos de construcción y promoción inmobiliaria deben ser entendido como un tejido donde las iniciativas de todos los actores (privados, estatales y comunitarios) y sus modalidades (formales e informales) forman parte de un conjunto donde se entrecruzan las lógicas del mercado, del Estado y de la necesidad, que sólo pueden ser entendidas si se ponen en claro sus articulaciones.

La posibilidad de ampliar diversos circuitos económicos y comerciales de alta rentabilidad representa una oportunidad para el despegue de la inversión privada, la ampliación de vínculos y redes de carácter exógeno y la consolidación del bloque comercial. Desde el punto estratégico, político económico, los puntos o áreas de mayor interés para el capital inmobiliario y comercial son los pertenecientes a los cinco corregimientos que presentan mayor densidad de población aunque su situación es vulnerable pero la ubicación en la zona central, la cual limita con el área canalera y el resto del corredor transístmico , considerados aéreas estratégicas por la gran cantidad de infraestructuras viales, carreteras, autopista y el metro, simboliza un desafío para los promotores que aspiran al control de los espacios para la explotación capitalista.

El nuevo modelo de acumulación emergente requiere con urgencia transformar los espacios deprimidos del distrito de San Miguelito y ponerlos al servicio de las bases productivas representada por el capital inmobiliario, y para el desarrollo de nuevas actividades (servicios avanzados terciarios, turismo, grandes superficies comerciales, logística, etc.).

El desmantelamiento de las estructuras originarias del distrito de San Miguelito carentes de planificación (pequeñas industria comercio y viviendas), parece ser inminente para darle paso al desarrollo de formas espaciales específicas para albergar actividades, tales como parques empresariales, grandes contenedores especializados (hipermercados, centros comerciales, centros de ocio, multicines, vivienda de costos que superan el cuarto de millón de dólares como mínimo etc.). 
Corriendo la misma suerte de los Tres espacios históricos, tres escalas y objetos a priori, completamente ajenos en su naturaleza, se convirtieron en punto de referencia para nuestra investigación: a) La transformación del régimen de propiedad y el uso de las tierras comunales en la Inglaterra rural de la transición del feudalismo al capitalismo; b) la evolución de los barrios populares en Manhattan durante la segunda mitad del siglo XIX; c) la reconfiguración de la comunidad obrera en Alemania y EE.UU. entre la Gran Depresión del último tercio del siglo XIX y la segunda guerra mundial. A pesar de su disparidad, la mirada ha caído sobre estos espacios y períodos históricos de forma natural por la densidad de lo que a primera vista podría parecer su único punto en común, una clara dimensión procesual y de profunda transformación en las formas de vida de la fuerza de trabajo.

a) Como primera evidencia presentamos la modificación del espacio que albergaba a las ciudades gremiales en Inglaterra, sustituidas por la formación de una nueva lógica territorial, en los enclaves rurales de producción doméstica y su alianza con la primera manufactura como espacios emergentes de la nueva economía, y la consolidación de las grandes ciudades mercantiles lo cual daría como resultado el surgimiento de un mercado nacional en formación y un mercado colonial en expansión.

b) Un segundo elemento que se debe considerar lo constituye, la reconfiguración integral de la repartición económica y social del espacio residencial de Manhattan, con un alto nivel y con conciencia de clase en sentido norte-sur , cedió su espacio surgido del auge económico de la ciudad a nuevas forma de explotación productiva estructurando de esta manera un nuevo tejido comercial e industrial y otros servicios públicos, modificando su espacio social.

C) Un tercer caso lo fue la reorganización integral del espacio de vida, de producción y de reproducción de los espacios de Berlín y Japón adecuados a 
los parámetros metropolitanos, siguiendo los patrones y recomendaciones para un nuevo esquema de desarrollo urbano.

Fenómeno advertido por Henri Lefevre en su obra El derecho a la ciudad en la que trataba la tendencia generalizada hacia la urbanización y reflexionaba sobre sus repercusiones para el ser humano y para el futuro de la humanidad. Lefebvre se refería al - derecho a la ciudad • como uno de los derechos fundamentales del ser humano y de la ciudadanía, un derecho que implica la motivación de la sociedad civil para recrear la ciudad como parte de una emisión común y colectiva el análisis de Lefebvre: la destrucción gradual del modelo de ciudad tradicional, el ascenso de un sociedad globalizada de la cual son expulsados, a diferentes velocidades y bajo aspectos diversos, los $\cdot$ marginados • .los excluidos de los beneficios de la globalización. Y la necesidad de que a nivel internacional se produzcan debates sobre ese derecho. Si el $\cdot$ derecho a la ciudad permite la posibilidad de múltiples interpretaciones, su persistencia subraya a su vez la importancia de las cuestiones sociales subyacentes que afectan, hoy más que nunca, a los debates sobre la urbanización y su papel en el futuro.

Es el hacinamiento urbano que da lugar a un tejido urbano ( Lefevre, 1972), un sistema de soportes materiales (Padilla, 1984) o un dispositivo espacial (Jaramillo, 2009), que resulta de la urdimbre de diferentes productos de la actividad de la construcción, cuya valor de uso complejo sólo se hace efectivo por su articulación especifica, mostrando la contradicción entre la lógica privada de la producción capitalista, que sólo vela por los efectos inmediatos de los resultados de su acción individual, fundamentalmente su rentabilidad, sin reparar en los impactos en el conjunto, que reclaman una y otra vez la necesidad de coordinación y control colectivo de la producción y uso de la ciudad (Abramo, 2011) para evitar los efectos perversos de diferente tipo (económicos, sociales, ambientales, urbanísticos) cuando se deja a su libre albedrio a las fuerzas del mercado, que explica la importancia de las presiones sociales para estimular la intervención reguladora del Estado en el desarrollo urbano. Dichos objetos constituyen el medio ambiente construido como resultado de productos, y 
procesos de producción (pasados y actuales) de una rama particular de la economía: la industria de la construcción o de formas menos institucionalizadas.

\section{Consideraciones Finales}

El modelo de desarrollo inmobiliario instaurado en Panamá a partir de los años ochenta ve en el distrito de San Miguelito el sitio oportuno para plasmar lo que resalta Manuel Castells (1995) cuando expresa: asistimos a una transformación estructural de la sociedad, porque la nuestra es ya una sociedad totalmente distinta a la industrial. De esta nueva sociedad, señaló, no podemos hacer aún un dibujo preciso ni establecer predicciones concretas sobre su evolución, pero sí que es una sociedad de redes. Recordó que actualmente la mitad de la población del planeta vive en entornos urbanos y que se prevé que a finales del presente siglo casi el ochenta por ciento de los seres humanos vivirá en ciudades.

Para Castells (1995), el ámbito geográfico que adquiere cada vez más relevancia son las regiones metropolitanas, formadas por constelaciones urbanas integradas funcional y socialmente interesantes, unidades metropolitanas cada vez más entrelazadas por infraestructuras físicas y virtuales. En su opinión, los sistemas de comunicación avanzados crean una nueva geografía, caracterizada por la concentración y a la vez por la descentralización, en este caso, argumenta, no son conceptos opuestos, sino que únicamente describen nuestra propia realidad; que estamos generando una arquitectura espacial de concentracióndescentralización, tanto en el entorno urbano como en los flujos de población y actividades. Ya no podemos hablar de centro y periferia sino de diferentes centros conectados entre si, el verdadero impacto de las telecomunicaciones es su capacidad de concentrar y descentralizar dentro de una misma concentración urbana.

Esta nueva arquitectura espacial comporta una transformación de las relaciones sociales, que también se convierten en duales, con una tendencia clara a la individualización, pero con un alto grado de comunalización, como lo 
demuestra el hecho de que nos refugiemos cada vez más en la cultura. Somos cada día más conscientes de nuestra individualidad, pero al mismo tiempo creamos nuestras propias redes de relación que nos permiten seguir conectados y compartir intereses aunque estemos aislados socialmente.

La crisis de la familia patriarcal provoca nuevas estructuras y nuevas necesidades, de vivienda, de transporte, etc. A este cambio de modelo familiar se suma, además, un cambio en las maneras de producir, del cual es síntoma más evidente es la aparición de las empresas en red, que "es la forma dominante en las empresas más dinámicas y competitivas de hoy en día" señaló Castells (1995). La actividad empresarial no se centra ya en una empresa, que puede continuar ejerciendo la gestión pero que necesita de otras empresas para llevar a cabo su proyecto empresarial. Eso comporta que los espacios de trabajo se diluyan y que la separación tradicional entre trabajo y familia ya no funcione.

Es evidente que estamos asistiendo a una ruptura de los patrones de comunicación, tanto entre culturas como entre personas, y ello provoca la segregación espacial "algo que es innegable en la última década en la que no es que se haya excluido a los pobres sino que se ha potenciado a los ricos" y la transformación de algunos espacios urbanos en "una especie de parques temáticos o ciudades para visitantes". Castells (1995) afirma que la sociedad actual se caracteriza por la inclusión en redes y la exclusión generada por la separación de espacios. Y que los movimientos sociales que la conforman están divididos entre aquellas comunidades locales "a la defensiva", que bloquean el desarrollo porque necesitan conservar lo que han logrado, y los movimientos ambientales vinculados a la ecología, que apuestan por un cambio social que mantenga el bienestar. $Y$ todo ello tiene consecuencias sobre la planificación y es a la vez su resultado. Por eso, es preciso repensar y adecuar el espacio público a todas estas dualidades.

Fenómeno apreciado con claridad en el caso del Distrito de San Miguelito, existen en Panamá, experiencias de aéreas o espacio deprimidos, transformados en 
centros de alto poder adquisitivo y excluyentes como Loma la Pava, Marañón, San Sebastián, Boca la Caja.

\section{Referencias Bibliograficas}

Álvaro Sevilla Urbanismo y reproducción social una introducción a su historia 2012

Castells, M. (1995). La ciudad informacional. Madrid: Alianza.

Castillo, Jorge A. (1986). Formación Social Panameña 1850-1960.

Castro, Guillermo. El agua entre dos mares). Editorial Panamá: Ciudad del Saber. 2010

Farnum C., F. y V. Murillo G. (2015). Biodiversidad y Aspectos Ecológicos de los Parches Boscoso al borde de la Carretera Boyd Roosevelt tramo PanamáColon. Revista Colón Ciencias, Tecnología y Negocios 2 (2): 49-63.

Habermas, J. 1985. "Conciencia moral y acción comunicativa". Península, Barcelona.

Kuhn T. La estructura de las revoluciones científicas. México: Fondo de Cultura Económica; 1971

Lefebvre H. (1974). La production de l'espace. París: Anthropos.

Marx, Carlos y Engel Federico La ideología alemana. Editorial Progreso, 1998.

Montoya, A. (1997). Historia de San Miguelito. Panamá: Editorial Portobelo.

Tejeira, Gil Blas 1962. Pueblos perdidos. Edición 2. Editor Litho-Impresora Panamá. 244 páginas.

Worster, Donald, 2006: Transformaciones de la Tierra. Ensayos de historia ambiental. Selección, traducción y presentación de Guillermo Castro H. Editorial Universidad Estatal a Distancia. Serie Educación Ambiental, No. 1. San José, Costa Rica. 